\title{
Filtering of Interferometric SAR Phase Images as a Fuzzy Matching-Pursuit Blind Estimation
}

\author{
Bruno Aiazzi \\ Institute of Applied Physics "Nello Carrara” (IFAC), National Research Council (CNR), Via Panciatichi 64, 50127 Florence, Italy
} Email: b.aiazzi@ifac.cnr.it

Stefano Baronti

Institute of Applied Physics "Nello Carrara" (IFAC), National Research Council (CNR), Via Panciatichi 64, 50127 Florence, Italy Email:s.baronti@ifac.cnr.it

\section{Massimo Bianchini}

Department of Electronics and Telecommunications (DET), University of Florence, Via di Santa Marta 3, 50139 Florence, Italy Email:mb@massimobianchini.it

\author{
Alessandro Mori \\ Department of Electronics and Telecommunications (DET), University of Florence, Via di Santa Marta 3, 50139 Florence, Italy \\ Email: mori@ing.unifi.it

\section{Luciano Alparone} \\ Department of Electronics and Telecommunications (DET), University of Florence, Via di Santa Marta 3, 50139 Florence, Italy \\ Email: alparone@lci.det.unifi.it
}

\section{Received 6 August 2004; Revised 6 April 2005}

\begin{abstract}
We present an original application of fuzzy logic to restoration of phase images from interferometric synthetic aperture radar (InSAR), which are affected by zero-mean uncorrelated noise, whose variance depends on the underlying coherence, thereby yielding a nonstationary random noise process. Spatial filtering of the phase noise is recommended, either before phase unwrapping is accomplished, or simultaneously with it. In fact, phase unwrapping basically relies on a smoothness constraint of the phase field, which is severely hampered by the noise. Space-varying linear MMSE estimation is stated as a problem of matching pursuit, in which the estimator is obtained as an expansion in series of a finite number of prototype estimators, fitting the spatial features of the different statistical classes encountered, for example, fringes and steep slope areas. Such estimators are calculated in a fuzzy fashion through an automatic training procedure. The space-varying coefficients of the expansion are stated as degrees of fuzzy membership of a pixel to each of the estimators. Neither a priori knowledge on the noise variance is required nor particular signal and noise models are assumed. Filtering performances on simulated phase images show a steady SNR improvement over conventional box filtering. Applications of the proposed filter to interferometric phase images demonstrate a superior ability of restoring fringes yet preserving their discontinuities, together with an effective noise smoothing performance, irrespective of locally varying coherence characteristics.
\end{abstract}

Keywords and phrases: adaptive filtering, blind estimation, coherence, fuzzy logic, interferometric synthetic aperture radar, phase noise filtering.

\section{INTRODUCTION}

Synthetic aperture radar (SAR) enables imaging of the Earth by processing microwave backscattering data collected along the flight path of an airborne or spaceborne platform. This results in high-resolution images of the local complex ground reflectivity. Given two SAR images obtained from slightly different flight paths, if the complex-valued pixels of one image are multiplied by the coregistered complex conjugate pixels of the other, then the phase of the resulting product image constitutes a SAR interferogram $[1,2]$. The significance of this phase image is that it contains information on terrain height. Proper processing yields the so-called digital elevation model (DEM), which represents the topography of the terrain. 
The key problem in obtaining the DEM lies in the fact that measured phase differences are given as a wrapped phase field of principal values with range $-\pi$ to $\pi$, thereby being undetermined within multiples of $2 \pi$. It is necessary to perform a $2 \mathrm{D}$ phase unwrapping operation $[3,4]$ to remove phase jumps between neighboring pixels larger than $\pi$ by adding or subtracting multiples of $2 \pi$, so that the resulting distribution can be considered as samples of an underlying smooth function [5]. A major challenge of $2 \mathrm{D}$ phase unwrapping stems from the fact that the noise corrupting the phase field, due to, for example, temporal and/or baseline decorrelation [6], introduces local inconsistencies of the data referred to as residues [7]. Any phase unwrapping algorithm is sensitive to the presence of residues which make contour integrations of phase gradient path-dependent [7]. Residues are the visible effect of discontinuities of the underlying (absolute, unknown) phase field on the wrapped phase, and derive from localized inconsistencies in the vector field obtained by wrapping the gradient of the wrapped phase field, that is, the instantaneous frequency [8]. Such inconsistencies make the latter field locally rotational, so that it cannot be considered as the gradient of a scalar field, that is, the absolute phase. To restore the previously mentioned "wrapped gradient" field, making it irrotational, and thus integrable to yield the absolute phase, residues have to be "corrected." This is achieved by introducing integer cycle additions to the estimated gradient [9]. What causes residues to occur on InSAR data is both noise, intended as local random errors in the phase estimation, which can be due, for example, to decorrelation or thermal sensor noise, as well as actual discontinuities in the underlying phase field [10]. These can be generated, for example, by strong topographic variations, or locally unfavorable geometric acquisition parameters (shadowing, layover, etc.).

Once the noise sensitivity of $2 \mathrm{D}$ phase unwrapping algorithms has been recognized, it would be useful to remove noise from the measured interferogram before processing it further. The main concern must be spatial resolution preservation and accuracy of the subsequent phase unwrapping step. Other requirements are, obviously, affordable computational complexity, robustness, and ease of use. Advantage of a preprocessing scheme is that existing phase unwrapping algorithms can be used without any modification.

Unless knowledge of the nonplanar scene topography is exploited for mitigating geometrical decorrelation effects [11], the most straightforward approach to interferogram noise filtering is to consider it as an image processing problem. That is, a noise-suppressing transformation is sought that maps real-valued images onto real-valued images. Such a transformation will be in general adaptive, that is, dependent on the data it operates on. From the nature of the differential phase data, it is clear that finding a transformation that can preserve resolution of the data and accuracy of the phase unwrapping is a hard task. The fringes (lines of discontinuity) in the data should not be affected with respect to their location, sharpness, and jump height, ruling out linear lowpass filtering techniques that may lead to severe distortion. Lee's refined filter [12], widely used for despeckling SAR reflectivity images with improved geometrical adaptivity and texture preservation, has been adjusted to filter out InSAR phase noise [13]. The latter exploits a $9 \times 9$ split window featuring 16 directional subwindows, to capture locally homogeneous neighborhoods in which a local-statistics filtering is performed. Following more recent approaches, it has been demonstrated that modeling of the second-order statistics of the phase image by using complex-valued Markov random fields (CMRFs) may help the subsequent unwrapping step [14]. MRFs have also been used for restoration of the phase field [15]. Furthermore, an interferogram typically exhibits structures on different scales due to varying fringe density. This suggests that multiresolution techniques might be profitably employed, for both phase denoising [16] and unwrapping [17].

The first-order statistics of the interferometric phase images have been characterized by a probability density function (PDF) based on the circular Gaussian assumption [18]. For noise reduction, multilook processing is frequently implemented by coherently averaging neighboring pixels [1]. It has been proven [19] that the multilook phase noise has a hypergeometric PDF and that the phase noise standard deviation depends on coherence $[20,21]$ and number of looks. More exactly, it is either a decreasing function of the coherence for a given number of looks, or a decreasing function of the number of looks for a given nonzero coherence. Furthermore, the actual number of independent looks is usually smaller than the number of samples averaged [22], for example, due to preprocessing of raw data before focusing. When coherence is null, the noise level is theoretically independent of the number of looks [19]. Thus, the noise level varies across the image in an inhomogeneous form, according to coherence values, thereby making model-based denoising a nontrivial problem.

Estimation of a noise-free image from its corrupted observation is usually achieved as a weighted combination of the output of two or more pixel estimators, for example, spatial average and pixel level itself, with weights summing to one and adaptively calculated from the noise model parameters and from the local statistics of the noisy image, in such a way to yield, for example, a minimum MSE (MMSE) estimate [23, 24]. MMSE estimate, however, is achieved to the extent by which the parametric noise model is known, and space-varying statistics are approximated by local sample statistics. Such an ergodicity assumption may not hold on edges, thereby leading to poor filtering.

In this work, the problem of phase noise reduction is approached as a blind estimation. According to this term, the underlying signal and noise models may be contextually learned and used to drive a model-based filtering. Alternatively, the denoising algorithm may not be based on a particular model, thereby overcoming the limitations of a model that is either unknown or crucial to estimate. Fuzzy logic provides useful tools to design a blind estimator. The proposed fuzzy matching-pursuit (FMP) filtering does not require "a priori" knowledge on the noise variance and model, either signal-dependent or not. Only the window sizes and number of prototype estimators must be specified. Besides its 


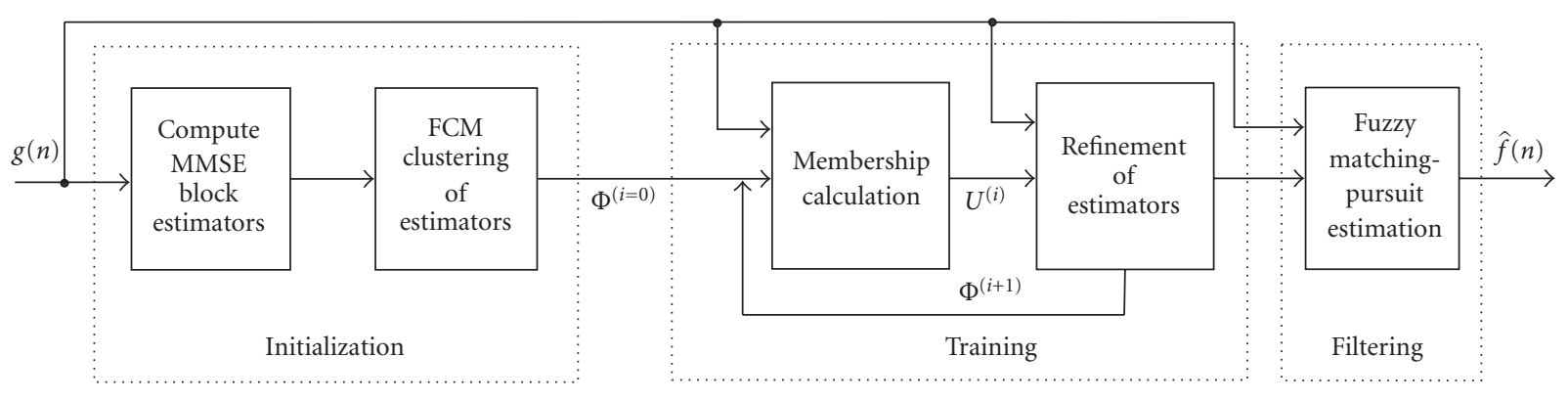

FIGURE 1: Flowchart of fuzzy matching-pursuit (FMP) adaptive filtering procedure.

outstanding performances—-both visual and SNR — for filtering of simulated noise [25], preliminary results of interferometric SAR (InSAR) phase noise filtering seem to be promising as well [26].

The paper is organized as follows. Section 2 describes the various steps of the procedure. Section 3 reports experimental results on simulated interferograms, varying with coherence level, and on a true InSAR phase image; advantages over conventional box filtering are demonstrated. Concluding remarks are drawn in Section 4.

\section{MATCHING-PURSUIT FILTERING SCHEME}

Matching pursuit (MP) is an iterative method to expand a signal by using an overcomplete dictionary of nonorthogonal functions [27]. Although MP has been recently employed mostly for video coding [28], its original formulation is quite general. Under this perspective, we assume that the unknown MMSE adaptive estimator at the current pixel position may be expressed as a series expansion of a "dictionary" made of a number of estimators fitting the different classes of spatial features occurring throughout the image. Given the ambiguous or "fuzzy" nature of the problem, the coefficients of the expansion are taken not as scalar products, as for the conventional formulation of MP [27], but as degrees of membership of that pixel to the estimators of the dictionary.

According to the flowchart shown in Figure 1, the filtering procedure can be summarized as follows. Images are first partitioned into square blocks, for example, $16 \times 16$, and an MMSE linear estimator is calculated for each block. From the large number of estimators obtained, a fuzzy clustering algorithm produces an initial guess of a user-specified number of prototype estimators that are delivered to an iterative procedure, in which firstly to each estimator pixels are given degrees of membership measuring the fitness of estimation. Then, estimators are recalculated from pixels depending on their degree of membership. The overall estimation will be fuzzy, being given by the sum of the output of each estimator weighted by the memberships of the current pixel to that estimator. The linearity of estimation makes it possible to state the above problem as an approximation of the unknown space-varying MMSE estimator at each pixel, achieved through its projection onto a set of nonorthogonal prototype estimators capable to embody the statistical properties of the data.
Let $g(n)=f(n)+v(n)$ denote a noisy observation of the noise-free image $f(n)$. The $v(n)$ is a noise term assumed to be zero-mean and spatially uncorrelated, yet possibly signaldependent and nonstationary. If $f$ is nonstationary, signal dependence of $v$ usually implies its nonstationarity. The variance of $v$ may be unknown and not straightforwardly measurable. The MMSE estimate of $f$ is the conditional expectation of the noise-free signal to the noisy observed signal [24], that is, $E[f \mid g]$. To derive a solution of the problem, assumptions are usually made on the noise model, as well as on the signal, for example, for a maximum a posteriori (MAP) estimation of speckle-free SAR reflectivity $[29,30]$.

In this work, a spatial estimation for the pixel $n$ is based on a linear combination of surrounding pixels lying within a noncausal square neighborhood of radius $R$ of the current pixel $n, \mathcal{N}_{\infty}^{R}(n)$, which does not comprise the pixel $n$. The subscript $\infty$ indicates a neighborhood of radius $R$ according to $L_{\infty}$ metrics. Thus, the estimation support has size $S=(2 R+1)^{2}-1$.

Let $\vec{\psi}(n)=\left\{\psi_{1}(n), \psi_{2}(n), \ldots, \psi_{S}(n)\right\}^{T}, \psi_{k}(n)=g(k)$, $k \in \mathcal{N}_{\infty}^{R}(n) \mid\|(k)-(n)\|_{2}=\delta_{k} \leq \delta_{k+1}$. The term $\delta_{k}$ indicates the distance of the $k$ th pixel to the center. Thus, $\vec{\psi}(n)$ denotes the vector containing the levels of the $S$ samples lying within $\mathcal{N}_{\infty}^{R}(n)$, sorted for increasing Euclidean distance from the current pixel $n$. On image edges, $\vec{\psi}(n)$ is padded with replicas of the nearest samples.

Let also $\vec{\phi}=\left\{\phi_{k} \in \mathbb{R}, k=1, \ldots, S\right\}^{T}\left(\sum_{k=1}^{S} \phi_{k}=1\right)$ denote the vector comprising the $S$ coefficients of a linear estimator operating on the support $\mathcal{N}_{\infty}^{R}(n)$. Thus, a linear estimation for $f(n)$ is defined as

$$
\hat{f}(n)=\sum_{k=1}^{S} \phi_{k} \cdot \psi_{k}(n)=\langle\vec{\phi}, \vec{\psi}(n)\rangle
$$

in which $\langle\cdot, \cdot\rangle$ indicates scalar (inner) product.

Since a unique linear estimator may be inadequate to the space-varying nature of the image data, a set of linear estimators is initialized, trained, and used to achieve a pixeladaptive estimation.

\subsection{Initialization}

The determination of the dictionary of estimators for the matching pursuit is the key to the success of the restoration process. It starts from observing that patterns of pixel values occurring within $\mathcal{N}_{\infty}^{R}(n)$ reflect local spatial features of 
the image, for example, edges, textures, and shadings. An efficient estimation should be capable to embody and reflect such features as much as possible.

After preliminarily partitioning the image into blocks of size $L \times L$, typically $8 \times 8$ or $16 \times 16$, a noncausal estimation support of size $S$ is set, and the $S$ coefficients of an MMSE linear estimator are calculated for each block by means of a standard least-squares (LS) algorithm [24]. Specifically, if $B$ denotes one block of the partition, the LS algorithm is given the pairs $\{(\vec{\psi}(n), g(n)) \mid n \in B\}$ to yield the related estimator $\vec{\phi}_{B}$. The rationale is that since $g(n)=f(n)+v(n)$ and the uncorrelated noise $v(n)$ cannot be predicted, the MMSE estimation of $g(n)$ will in principle coincide with $f(n)$.

The above process produces a large number of estimators, each optimized for a single block. The $S$ coefficients of each estimator are arranged into an $S$-dimensional space. More exactly, since the coefficients of any estimator sum to one, all estimators lie on the hyperplane passing through the unity-norm vectors of the coordinate axes. It can be noticed that statistically similar blocks exhibit similar estimators. Thus, the estimators found previously tend to cluster on the hyperplane, instead of being uniformly spread.

A user-provided number $M$ of representative estimators is identified by a fuzzy clustering procedure. Such "dominant" estimators are calculated as centroids of as many clusters in the estimators space, according to a vector Euclidean metric. Bezdek's fuzzy C means (FCM) algorithm $[31,32]$ was used (with exponent $m=1.1$ ) because it yields centroids that speed up convergence of the subsequent training.

Thus, an $S \times M$ matrix $\Phi^{(0)}=\left\{\vec{\phi}_{m}^{(0)}, m=1, \ldots, M\right\}$ containing the coefficients of the $M$ estimators is produced. The superscript (0) highlights that such estimators are startup values of the iterative refinement procedure which will yield the "dictionary" of estimators for matching pursuit.

\subsection{Training of estimators}

The $M$ estimators found out through fuzzy clustering are used to initialize an iterative training procedure in which firstly pixels are given degrees of membership to the estimators. Then, each estimator is recalculated based only on those pixels whose membership to it exceeds a specified threshold $\mu$. The above procedure is analogous to a relaxation labeling [33], in which the labeling is not crisp but fuzzy.

\subsubsection{Membership function}

The choice of the membership function is extremely crucial for the performance. Such a function should reflect the ability of an estimator to yield an estimate $\hat{f}(n)$ as close as possible, apart from the unpredictable white noise term $v(n)$, to $g(n)$ that is as close as possible to $f(n), v(n)$ having zero mean. A suitable fuzzy membership function of the $n$th pixel to the $m$ th estimator was devised as the reciprocal of the weighted squared estimation error, produced by the $m$ th estimator on a noncausal neighborhood of $n$, raised to an empirical exponent $\gamma$, and incremented by one to avoid divisions by zero. The noncausal neighborhood adopted is still square and, thus, uniquely defined by its radius $R^{\prime}$ in $L_{\infty}$ metrics.
$\mathcal{M}_{R^{\prime}} \triangleq \mathcal{N}_{\infty}^{R^{\prime}}$ will be referred to as membership support in the following. The weighted squared estimation error produced by the $m$ th estimator on the $n$th pixel is defined as

$$
\bar{d}_{m}^{2}(n)=\frac{\sum_{k \in \mathcal{M}_{R^{\prime}}(n)} \delta_{k}^{-1} \cdot\left[g(k)-\left\langle\vec{\phi}_{m}, \vec{\psi}(k)\right\rangle\right]^{2}}{\sum_{k \in \mathcal{M}_{R^{\prime}}} \delta_{k}^{-1}} .
$$

The weight of each squared estimation error is taken to be inversely proportional to the distance $\delta_{k}$ from the current pixel $n$. Thus, closer neighbors will contribute more than farther ones. The weighted squared error (2) is normalized to the sum of its weights. Thus, its magnitude is roughly independent of the neighborhood size.

The membership of the $n$th pixel to the $m$ th estimator will be

$$
U_{m}(n)=\frac{1}{1+\left[\bar{d}_{m}^{2}(n)\right]^{\gamma}} .
$$

As a matter of fact, (3) measures the capability of $\vec{\phi}_{m}$ to estimate the gray levels of the closest neighbors of the current pixel $n$. It can be inferred as the ability of $\vec{\phi}_{m}$ to estimate $f(n)$, that is, to yield $E[f(n) \mid g(k)], k \in \mathcal{N}_{\infty}^{R}$. If the outputs of the $m$ th estimator exactly fit the gray levels within the membership support of $n$, then $\bar{d}_{m}^{2}(n)$ will be zero, and hence $U_{m}(n)=1$. The membership exponent $\gamma$ rules the degree of fuzziness of the membership function; it is adjusted empirically and the value $\gamma=2$ will be used throughout.

Since the fuzzy membership will be used to measure a projection, same as a scalar product, the absolute membership given by (3) is normalized to yield a relative membership

$$
\tilde{U}_{m}(n)=\frac{U_{m}(n)}{\sum_{m=1}^{M} U_{m}(n)}
$$

suitable for a probabilistic clustering.

\subsubsection{Iterative refinement of estimators}

Step 0. For each pixel $n$, calculate initial membership array, $\tilde{U}_{m}^{(0)}(n), m=1, \ldots, M$, from initial set of estimators $\Phi^{(0)}=$ $\left\{\vec{\phi}_{m}^{(0)}, m=1, \ldots, M\right\}$ by using (2), (3), and (4); set iteration step $i=0$ and membership threshold $\mu$.

Step 1. Recalculate estimators $\left\{\vec{\phi}_{m}^{(i+1)}, m=1, \ldots, M\right\}$ from those pixels whose membership $\tilde{U}_{m}^{(i)}(n)$ exceeds $\mu$; weight by $\tilde{U}_{m}^{(i)}(n)$ the contribution of the pair of values $(\vec{\psi}(n), g(n))$ to $\vec{\phi}_{m}^{(i+1)}$ in the LS algorithm.

Step 2. For all pixel $n$, calculate memberships to estimators, $\tilde{U}_{m}^{(i+1)}(n), m=1, \ldots, M$.

Step 3. Convergence check; if OK, stop; else, increment $i$ by 1 and go to Step 1.

Convergence is checked by thresholding the MSE between the current estimated image and the estimated image at the previous iteration. The standard LS algorithm has been modified to account for the memberships of pixels to 


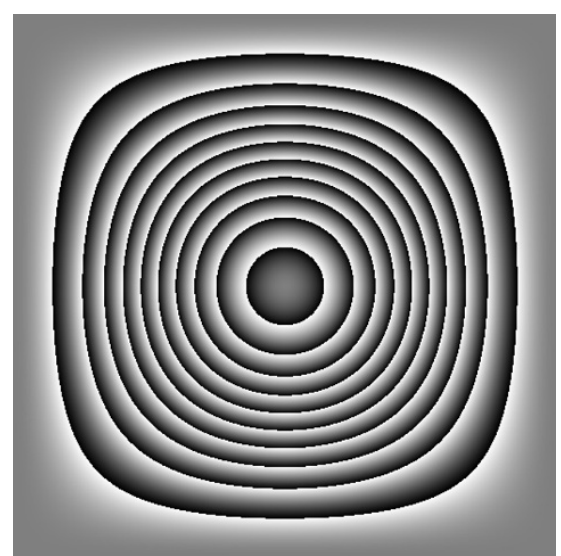

(a)

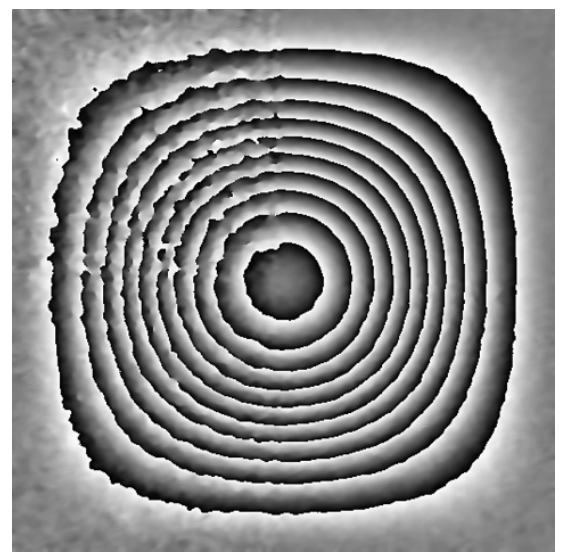

(c)

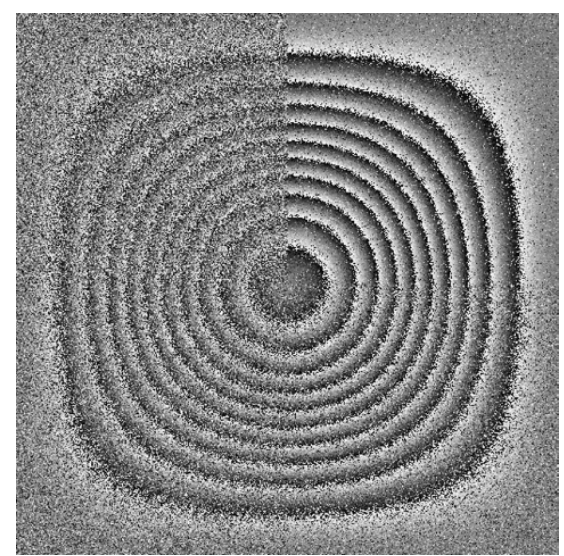

(b)

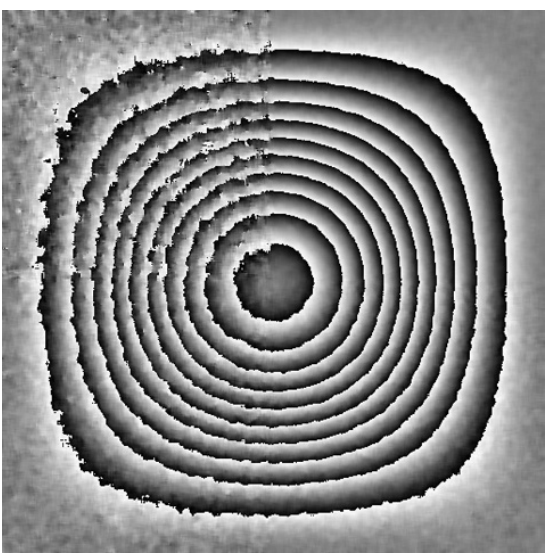

(d)

FIGURE 2: Filtering tests on simulated noisy interferograms: (a) original wrapped phase map $(512 \times 512)$; (b) one-look noisy interferogram, with coherence values $0.3,0.5,0.7,0.9$, counter-clockwise from top left quadrant; (c) $7 \times 7$ FMP filtering; and (d) $7 \times 7$ box filtering.

estimators at the previous iteration. Pixels having larger degrees of memberships to an estimator will contribute to that estimator more than pixels having smaller degrees. Furthermore, depending on the threshold $\mu$, a pixel may contribute, though to different extents, to more estimators, in the fuzzylogic spirit. The membership threshold $\mu$ is noncrucial for performances. The value $\mu=0.1$ will be used throughout.

\subsection{Fuzzy matching-pursuit estimation}

By the linearity of estimation, a weighted sum of the outputs of estimators is equal to the output of a linear combination of the same estimators with the same weights, that is, to calculate an adaptive estimator at every pixel,

$$
\vec{\phi}(n) \triangleq \sum_{m=1}^{M} \widetilde{U}_{m}(n) \cdot \vec{\phi}_{m}
$$

in which the weights are still provided by $\tilde{U}_{m}(n)$, that is, (4), with (2) calculated from the estimators $\left\{\vec{\phi}_{m}, m=1, \ldots, M\right\}$ after the last iteration stage. The estimator (5) yields the adaptive linear estimation as $\hat{f}(n)=\langle\vec{\phi}(n), \vec{\psi}(n)\rangle$. Equivalently, each pixel value $f(n)$ can be estimated as a soft switching, that is, a blending of the outputs of all the estimators, which are defined as

$$
\hat{f}_{m}(n)=\left\langle\vec{\phi}_{m}, \vec{\psi}(n)\right\rangle
$$

with the fuzzy estimation, $\hat{f}(n)$, given by

$$
\hat{f}(n)=\sum_{m=1}^{M} \tilde{U}_{m}(n) \cdot \hat{f}_{m}(n) .
$$

\section{EXPERIMENTAL RESULTS}

\subsection{Simulated InSAR phase images}

One-look interferograms have been generated starting from two phase maps, having different slopes, and white Gaussian variables cross-correlated by the desired amount of coherence, following the procedure described in [7]. Such interferograms exhibit either broad fringes (10 phase jumps) or tight fringes (20 jumps). The latter are obviously more critical for what concerns phase restoration. The original phase map with tight fringes and a mosaic of the related noisy phase maps, with coherence varying in $[0.3,0.9]$ at steps of 0.2 , are portrayed in Figures $2 \mathrm{a}$ and $2 \mathrm{~b}$, respectively. Noise level is inversely related to coherence values, as expected. 
TABLE 1: MSE between original noise-free wrapped phase map (with broad fringes) and filtered one-look interferograms, varying with processing window size and coherence $\left|\rho_{c}\right|$.

\begin{tabular}{l|ccccc}
\hline Broad fringes & $\left|\rho_{c}\right|=0.9$ & $\left|\rho_{c}\right|=0.7$ & $\left|\rho_{c}\right|=0.5$ & $\left|\rho_{c}\right|=0.3$ & Average \\
\hline FMP $(3 \times 3)$ & 0.0064 & 0.0202 & 0.0599 & 0.2103 & 0.0742 \\
FMP $(5 \times 5)$ & 0.0058 & 0.0184 & 0.0541 & 0.1861 & 0.0661 \\
FMP $(7 \times 7)$ & 0.0043 & 0.0121 & 0.0328 & 0.1017 & 0.0377 \\
Box $(3 \times 3)$ & 0.0374 & 0.1417 & 0.4710 & 1.1774 & 0.4565 \\
Box $(5 \times 5)$ & 0.0129 & 0.0431 & 0.1353 & 0.5104 & 0.1754 \\
Box $(7 \times 7)$ & 0.0066 & 0.0218 & 0.0642 & 0.2361 & 0.0822 \\
Unfiltered & 0.4859 & 1.1735 & 1.7809 & 2.3602 & 1.4481 \\
\hline
\end{tabular}

TABLE 2: MSE between original noise-free wrapped phase map (with tight fringes) and filtered one-look interferograms, varying with processing window size and coherence $\left|\rho_{c}\right|$.

\begin{tabular}{l|ccccc}
\hline Tight fringes & $\left|\rho_{c}\right|=0.9$ & $\left|\rho_{c}\right|=0.7$ & $\left|\rho_{c}\right|=0.5$ & $\left|\rho_{c}\right|=0.3$ & Average \\
\hline FMP $(3 \times 3)$ & 0.0147 & 0.0488 & 0.1582 & 0.5807 & 0.2004 \\
FMP $(5 \times 5)$ & 0.0080 & 0.0254 & 0.0726 & 0.2676 & 0.0932 \\
FMP $(7 \times 7)$ & 0.0083 & 0.0238 & 0.0608 & 0.2015 & 0.0735 \\
Box $(3 \times 3)$ & 0.0390 & 0.1500 & 0.4742 & 1.2218 & 0.4713 \\
Box $(5 \times 5)$ & 0.0140 & 0.0470 & 0.1478 & 0.5712 & 0.1948 \\
Box $(7 \times 7)$ & 0.0078 & 0.0259 & 0.0784 & 0.3029 & 0.1036 \\
Unfiltered & 0.4861 & 1.1736 & 1.7780 & 2.3790 & 1.4526 \\
\hline
\end{tabular}

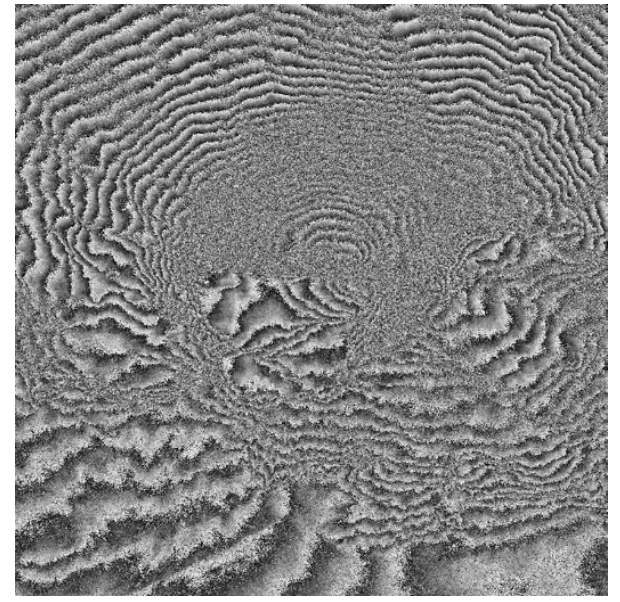

FIgURe 3: Topographic fringes of Mount Etna, Italy, from ERS-1/2 Tandem Mission: original $(1024 \times 1024$, 4-look coherent average along azimuth of complex interferogram).

Spatial filtering was applied to denoise the phase map. Following an established practice, the phase was filtered in its complex form, that is, $e^{j \phi}$ instead of $\phi$, to avoid $2 \pi$ phase jumps. FMP runs on windows of size $3 \times 3$ to $7 \times 7$, that is, $R=1,2,3$, with membership radius $R^{\prime}=\max \{(R-1), 1\}$ and either 8 or 16 prototype estimators. The initial block size $L \times L$ was set equal to $16 \times 16$. One iteration of estimators refinement was performed. An $n \times n$ box filtering $(n \times n$ spatial average) of the complex interferogram, being blind same as FMP, was chosen as benchmark algorithm for quantitative comparisons. Window sizes $3 \times 3$ to $7 \times 7$ were used.

Tables 1 and 2 report errors between original wrapped phase map and filtered mosaic interferograms, for the cases of broad and tight fringes, respectively. MSEs are separately calculated on the four quadrants to highlight trends varying with coherence. Unlike FMP, the window size of box filter is crucial: $3 \times 3$ is not adequate to effectively denoise the phase maps; $7 \times 7$ box filtering represents the best tradeoff and is displayed in Figure 2d. Results with window sizes larger than $7 \times 7$ are not reported: a $9 \times 9$ window leads to oversmoothing, which is accompanied by large geometric distortions in locations of phase jumps, particularly visible in the map with tightly packed fringes. Conversely, the restoration capability of FMP filter is less sensitive to the window size than that of box filtering, especially for the simulated map with broad fringes. A $7 \times 7$ window trades off smoothness and geometric distortion for FMP as well. The filtered phase map is displayed in Figure 2c. The average gain of $7 \times 7$ FMP filtering over $7 \times 7$ box filtering, whose results are shown in Figure 2d, is $1.5 \mathrm{~dB}$ for the case of tight fringes, more than $3 \mathrm{~dB}$ for the less crucial case of broad fringes.

Also residues have been calculated on the interferograms shown in Figures $2 \mathrm{c}$ and $2 \mathrm{~d}$. The majority of residues are concentrated in the noisiest quadrant, in which, however, their percentage is $0.14 \%$ for FMP and $0.55 \%$ for box filtering (both $7 \times 7$ ). Such low percentages are not surprising for interferograms simulating a highly regular topography, since they are due to the noise only.

\subsection{True InSAR phase images}

A topographic interferogram of Mount Etna, in Sicily, Italy, was produced from two ERS-1/2 SLC tandem images acquired on consecutive days. The baseline length is $140 \mathrm{~m}$. Four consecutive samples along azimuth of the interferogram have been coherently averaged to yield the multilook topographic phase map, shown in Figure 3. The highly 


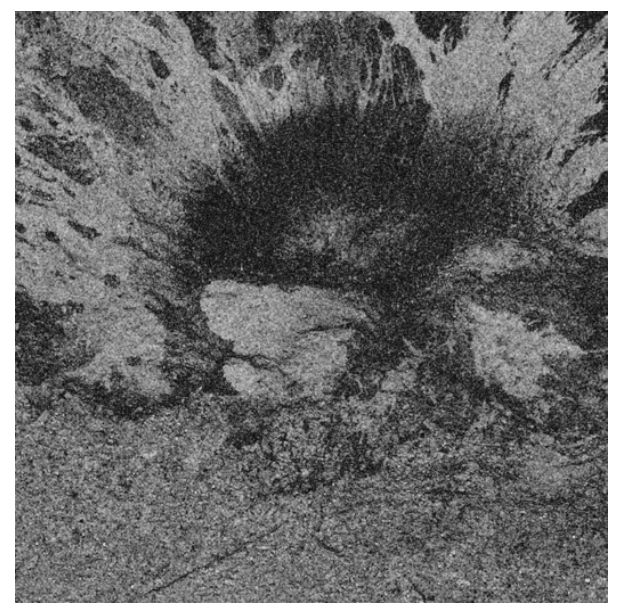

FIGURE 4: Tandem observations of Mount Etna: $1024 \times 1024$, 4-look coherence map.

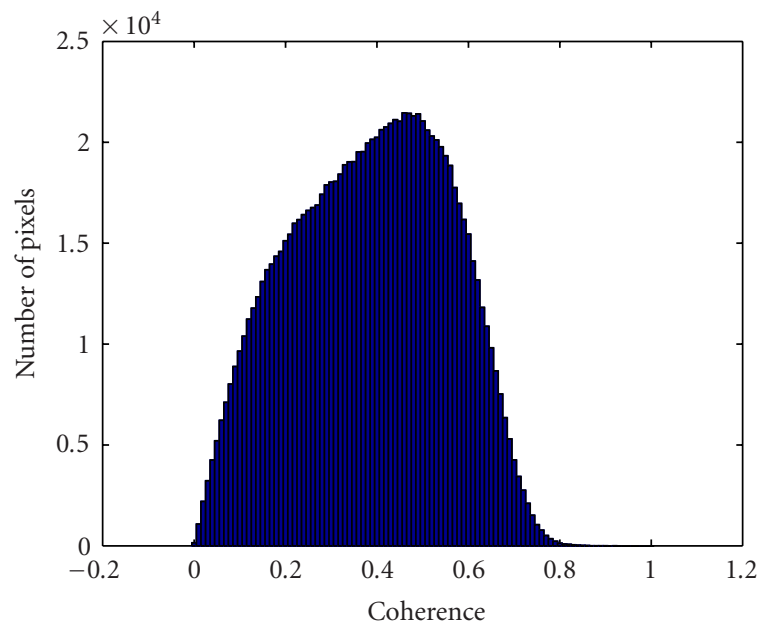

FIgURE 5: Tandem observations of Mount Etna: 4-look coherence histogram.

space-varying noisiness of the phase data stands out: on the upper side of the volcano, near the craters, the SNR is so low that fringes almost vanish, up to completely disappear.

The coherence map estimated on a $3 \times 3$ sliding window of the 4-look complex interferogram is shown in Figure 4. Coherence varies from 0.001 to 0.922 , with average 0.385 ; its histogram, shown in Figure 5, highlights the presence of two dominant high- and low-coherence classes, whose distributions are partially overlapped. Low-coherence pixels are mostly concentrated on the upper side of the crater and are due to low echo power and shadowing effects.

The $5 \times 5$ filtered image is shown in Figure 6 as best visual result of FMP. The noise is carefully removed and fringes appear sharp and clean in high SNR areas. However, in the low-coherence area above the craters, low SNR fringes are carefully restored, regardless of their rate. The

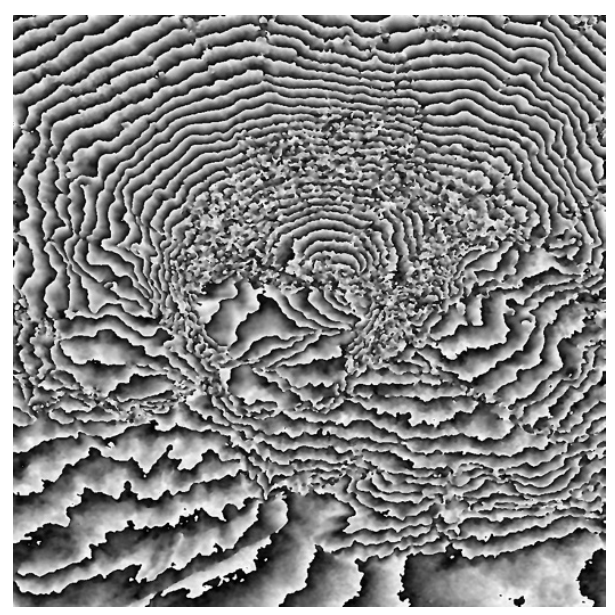

FIGURE 6: $1024 \times 1024$ map of 4-look topographic phase processed by FMP $(5 \times 5$ neighborhood and 8 prototype estimators $)$ in complex exponential form.

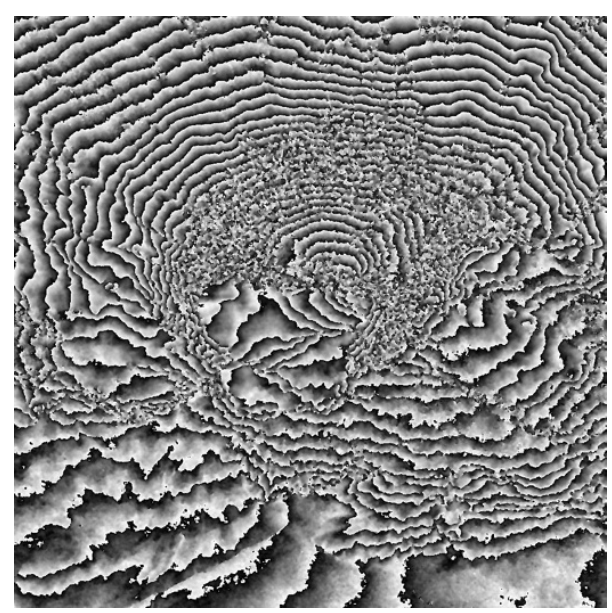

FIGURE 7: $1024 \times 1024$ map of 4-look topographic phase processed by $5 \times 5$ box filtering in complex exponential form.

window size smaller than that used for one-look simulated data depends on the different number of looks of the true data.

Compared to FMP filtering with same window size, the filtered map produced by a $5 \times 5$ box filter is somewhat noisier, especially on fringe edges and low-coherence areas. The difference (modulo $2 \pi$ ) between raw and filtered phase maps was calculated and analyzed. The mean squared difference is reported in Table 3 for the different filters and window sizes. Although the trend obviously reveals that larger windows yield greater differences, $3 \times 3$ FMP removes an amount of what is expected to be noise, greater than $3 \times 3$ box filtering does, while $7 \times 7$ FMP removes less "noise" than $7 \times 7$ box; a $5 \times 5$ window yields comparable variance reduction. However, the $5 \times 5$ box-filtered map is visually noisier than the $5 \times 5$ FMP filtered map, see Figures 7 and 6 , respectively, 
$\mathrm{nz}=188220$

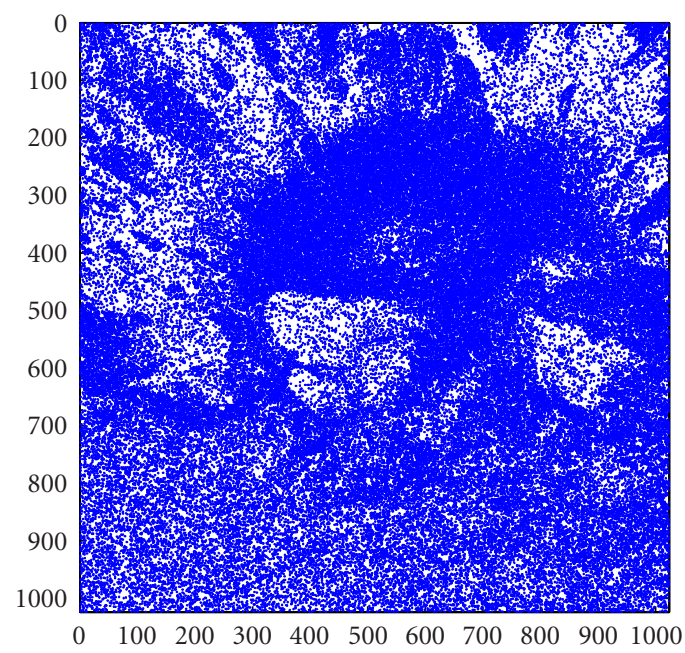

(a)

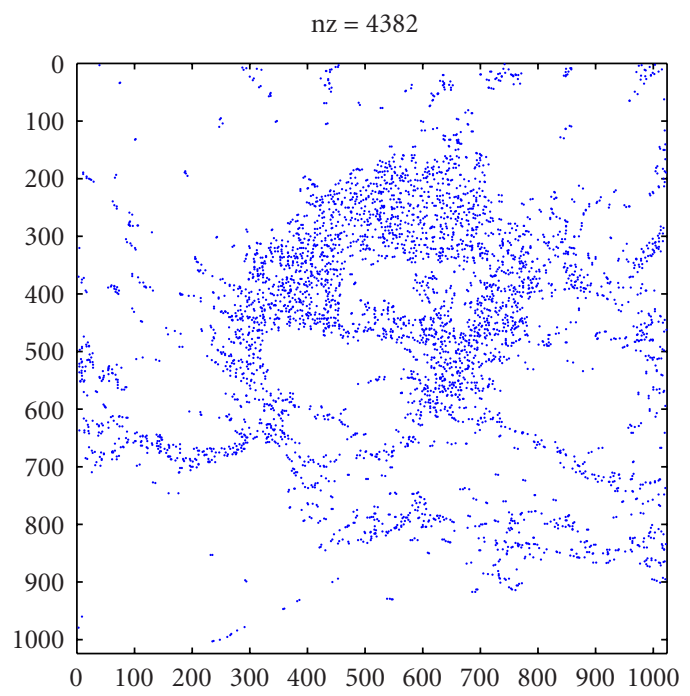

(c)

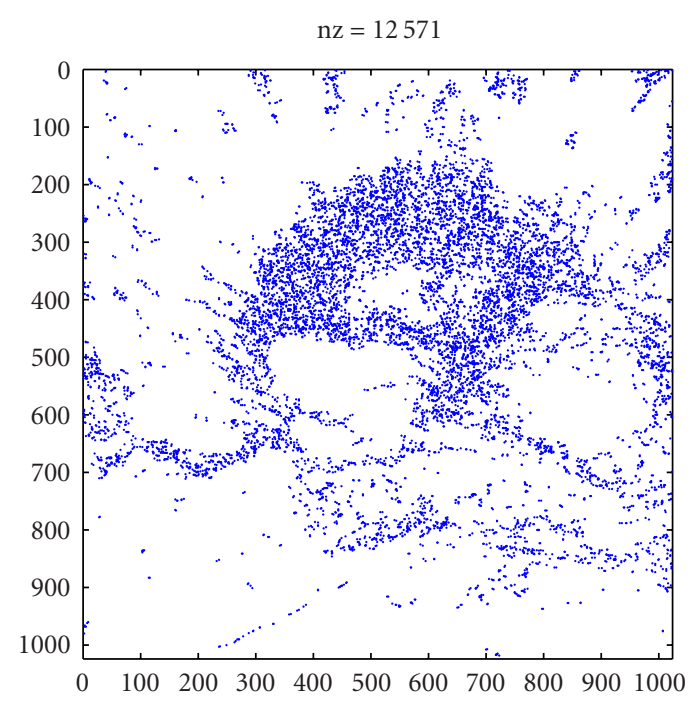

(b)

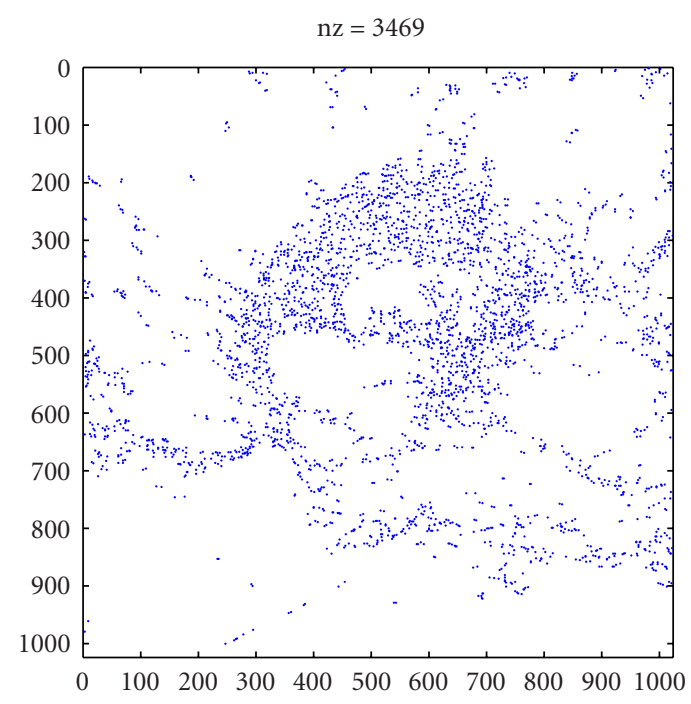

(d)

FIGURE 8: Residues of interferometric phase calculated from original and processed phase maps $(1024 \times 1024)$ : (a) original, (b) $5 \times 5$ box filtering, (c) $5 \times 5$ FMP filtering, and (d) $7 \times 7$ FMP filtering. Number of residues, nz, is reported for each map.

TABLE 3: MSE between raw and filtered phase maps varying with processing window size.

\begin{tabular}{c|ccc}
\hline Processing & $3 \times 3$ & $5 \times 5$ & $7 \times 7$ \\
\hline FMP & 1.3975 & 1.5153 & 1.5516 \\
Box & 1.3642 & 1.5285 & 1.6083 \\
\hline
\end{tabular}

though its MSE in Table 3 is slightly greater. Indeed, $5 \times 5$ box filtering leaves low-coherence areas "noisier" than FMP filtering of same size does and oversmoothes high-coherence areas. Conversely, $7 \times 7$ box filtering is capable of smoothing low-coherence areas, but largely distorts fringe edges, thereby resulting in a greater difference variance.

An analysis of residues has been carried out also on the Mount Etna interferograms. Table 4 highlights that, for
TABle 4: Percentages of residues of raw and processed phase data, varying with window size.

\begin{tabular}{c|lll}
\hline Processing & $3 \times 3$ & $5 \times 5$ & $7 \times 7$ \\
\hline Raw & 17.95 & 17.95 & 17.95 \\
Box & 2.67 & 1.20 & 0.80 \\
FMP & 0.82 & 0.42 & 0.33 \\
\hline
\end{tabular}

same window size, FMP filtering yields about three times less residues than box filtering does. This value is congruent with that obtained on simulated data, on which FMP yields four times less residues than a box filter of same size. Unlike the simulated case, for true InSAR data, residues are also originated by topography, which influences the more powerful FMP algorithm as well. Maps of residues are displayed in Figure 8. Their analysis reveals that the density of 
residues is inversely related to coherence. After processing, high-coherence areas are practically free of residues, while crucial very low-coherence areas are cleaned from residues much better by FMP than by box filtering, both $5 \times 5 ; 7 \times 7$ FMP can further reduce the percentage of residues from $0.42 \%$ to $0.33 \%$, at the cost of a slight oversmoothing of the phase map in high-coherence areas.

Eventually, the blind phase estimation carried out by both FMP and box filtering has the advantage that processing is not optimized for a particular signal and/or noise model; hence, filtering performances steadily follow the local SNR. However, FMP exhibits a subtler capability to yield consistent phase fields regardless of SNR; its behavior is moderately dependent on the window size, which is crucial for box filtering.

\section{CONCLUDING REMARKS}

Blind estimation of noise-free images from noisy observations was addressed as a problem of matching pursuit. Neither signal nor noise models are required, thanks to an iterative training of the dictionary of estimators which is driven by fuzzy rules. The subsequent fuzzy estimation guarantees a complete insensitiveness to the noise model and intensity. Application of FMP to InSAR topographic phase noise filtering yields encouraging results. Fringe-preserving smoothing and restoration is achieved even in the presence of a high fringe rate, regardless the local coherence characteristics ruling the space-varying noisiness of the phase field. Computational costs are more than affordable (about one minute for a $1024 \times 1024$ map on a standard Linux PC with $5 \times 5$ window and 8 estimators), though linearly increasing with size and number of estimators. Tests with 16 estimators have produced maps visually identical to the case of 8 estimators reported above, with only a slightly lower percentages of residues, thereby revealing that the number of prototype estimators is noncrucial, because their outputs are blended based on the fuzzy memberships of each pixel.

\section{ACKNOWLEDGMENTS}

The authors are grateful to G. Fornaro of IREA-CNR in Naples, Italy, for kindly providing the ERS data and for valuable discussions throughout. This research was carried out partly under Grants of the Italian Space Agency (ASI).

\section{REFERENCES}

[1] G. Franceschetti and G. Fornaro, "SAR interferometry," in Synthetic Aperture Radar Processing, G. Franceschetti and R. Lanari, Eds., chapter 4, pp. 167-223, CRC Press, New York, NY, USA, 1999.

[2] P. A. Rosen, S. Hensley, I. R. Joughin, et al., "Synthetic aperture radar interferometry," Proc. IEEE, vol. 88, no. 3, pp. 333$382,2000$.

[3] R. M. Goldstein, H. A. Zebker, and C. L. Werner, "Satellite radar interferometry: two-dimensional phase unwrapping," Radio Science, vol. 23, no. 4, pp. 713-720, 1988.
[4] D. C. Ghiglia and L. A. Romero, "Robust two-dimensional weighted and unweighted phase unwrapping that uses fast transforms and iterative methods," Journal of the Optical Society of America A, vol. 11, no. 1, pp. 107-117, 1994.

[5] G. Nico, G. Palubinskas, and M. Datcu, "Bayesian approaches to phase unwrapping: theoretical study," IEEE Trans. Signal Processing, vol. 48, no. 9, pp. 2545-2556, 2000.

[6] H. A. Zebker and J. Villasenor, "Decorrelation in interferometric radar echoes," IEEE Trans. Geosci. Remote Sensing, vol. 30, no. 5, pp. 950-959, 1992.

[7] D. C. Ghiglia and M. D. Pritt, Two-Dimensional Phase Unwrapping: Theory, Algorithms, and Software, John Wiley \& Sons, New York, NY, USA, 1998.

[8] U. Spagnolini, "2-D phase unwrapping and instantaneous frequency estimation," IEEE Trans. Geosci. Remote Sensing, vol. 33, no. 3, pp. 579-589, 1995.

[9] S. Stramaglia, A. Refice, and L. Guerriero, "Statistical mechanics approach to the phase unwrapping problem," Physica A: Statistical Mechanics and its Applications, vol. 276, no. 3-4, pp. 521-534, 2000.

[10] E. Trouvé, J.-M. Nicolas, and H. Maître, "Improving phase unwrapping techniques by the use of local frequency estimates," IEEE Trans. Geosci. Remote Sensing, vol. 36, no. 6, pp. 1963-1972, 1998.

[11] G. Fornaro and A. Monti-Guarnieri, "Minimum mean square error space-varying filtering of interferometric SAR data," IEEE Trans. Geosci. Remote Sensing, vol. 40, no. 1, pp. 11-21, 2002.

[12] J.-S. Lee, "Refined filtering of image noise using local statistics," Computer Vision, Graphics, and Image Processing, vol. 15, no. 2, pp. 380-389, 1981.

[13] J.-S. Lee, K. P. Papathanassiou, T. L. Ainsworth, M. R. Grunes, and A. Reigber, "A new technique for noise filtering of SAR interferometric phase images," IEEE Trans. Geosci. Remote Sensing, vol. 36, no. 5, pp. 1456-1465, 1998.

[14] A. B. Suksmono and A. Hirose, "Adaptive noise reduction of InSAR images based on a complex-valued MRF model and its application to phase unwrapping problem," IEEE Trans. Geosci. Remote Sensing, vol. 40, no. 3, pp. 699-709, 2002.

[15] G. Ferraiuolo and G. Poggi, "A Bayesian filtering technique for SAR interferometric phase fields," IEEE Trans. Image Processing, vol. 13, no. 10, pp. 1368-1378, 2004.

[16] C. Lopéz-Martínez and X. Fàbregas, "Modeling and reduction of SAR interferometric phase noise in the wavelet domain," IEEE Trans. Geosci. Remote Sensing, vol. 40, no. 12, pp. 25532566, 2002.

[17] G. W. Davidson and R. Bamler, "Multiresolution phase unwrapping for SAR interferometry," IEEE Trans. Geosci. Remote Sensing, vol. 37, no. 1, pp. 163-174, 1999.

[18] C. Oliver and S. Quegan, Understanding Synthetic Aperture Radar Images, Artech House, Boston, Mass, USA, 1998.

[19] J.-S. Lee, K. W. Hoppel, S. A. Mango, and A. R. Miller, "Intensity and phase statistics of multilook polarimetric and interferometric SAR imagery," IEEE Trans. Geosci. Remote Sensing, vol. 32, no. 5, pp. 1017-1028, 1994.

[20] R. Touzi, A. Lopès, J. Bruniquel, and P. W. Vachon, "Coherence estimation for SAR imagery," IEEE Trans. Geosci. Remote Sensing, vol. 37, no. 1, pp. 135-149, 1999.

[21] B. Aiazzi, L. Alparone, S. Baronti, and A. Garzelli, "Coherence estimation from multilook incoherent SAR imagery," IEEE Trans. Geosci. Remote Sensing, vol. 41, no. 11, pp. 2531-2539, 2003.

[22] C. H. Gierull and I. C. Sikaneta, "Estimating the effective number of looks in interferometric SAR data," IEEE 
Trans. Geosci. Remote Sensing, vol. 40, no. 8, pp. 1733-1742, 2002.

[23] D. T. Kuan, A. A. Sawchuk, T. C. Strand, and P. Chavel, "Adaptive noise smoothing filter for images with signal-dependent noise," IEEE Trans. Pattern Anal. Machine Intell., vol. 7, no. 2, pp. 165-177, 1985.

[24] S. M. Kay, Fundamentals of Statistical Processing, Volume I: Estimation Theory, Prentice-Hall, Englewood Cliffs, NJ, USA, 1993.

[25] B. Aiazzi, S. Baronti, and L. Alparone, "Blind image estimation through fuzzy matching pursuits," in Proc. IEEE International Conference on Image Processing (ICIP '01), vol. 1, pp. 241-244, Thessaloniki, Greece, October 2001.

[26] B. Aiazzi, L. Alparone, S. Baronti, and M. Bianchini, "Blind estimation of interferometric SAR phase images through fuzzy matching-pursuits," in Image and Signal Processing for Remote Sensing VII, S. B. Serpico, Ed., vol. 4541 of Proceedings of SPIE, pp. 59-69, Toulouse, France, September 2001.

[27] S. G. Mallat and Z. Zhang, "Matching pursuits with timefrequency dictionaries," IEEE Trans. Signal Processing, vol. 41, no. 12, pp. 3397-3415, 1993.

[28] R. Neff and A. Zakhor, "Very low bit-rate video coding based on matching pursuits," IEEE Trans. Circuits Syst. Video Technol., vol. 7, no. 1, pp. 158-171, 1997.

[29] A. Lopès, E. Nezry, R. Touzi, and H. Laur, "Structure detection and statistical adaptive speckle filtering in SAR images," International Journal of Remote Sensing, vol. 14, no. 9, pp. 17351758, 1993.

[30] S. Foucher, G. B. Bénié, and J.-M. Boucher, "Multiscale MAP filtering of SAR images," IEEE Trans. Image Processing, vol. 10, no. 1, pp. 49-60, 2001.

[31] A. Baraldi and P. Blonda, "A survey of fuzzy clustering algorithms for pattern recognition. I," IEEE Trans. Syst., Man, Cybern. B, vol. 29, no. 6, pp. 778-785, 1999.

[32] A. Baraldi and P. Blonda, "A survey of fuzzy clustering algorithms for pattern recognition. II," IEEE Trans. Syst., Man, Cybern. B, vol. 29, no. 6, pp. 786-801, 1999.

[33] K. Fukunaga, Introduction to Statistical Pattern Recognition, Academic Press, Boston, Mass, USA, 2nd edition, 1990.

Bruno Aiazzi was born in Borgo San Lorenzo, Florence, Italy, in 1961. He received the Laurea degree in electronic engineering from the University of Florence, Florence, Italy, in 1991. In 1992, he won a Fellowship on digital image compression for broadband communications networks, supported by the National Research Council (CNR), Italy. After several years as a Research Fellow and Research Assistant, in

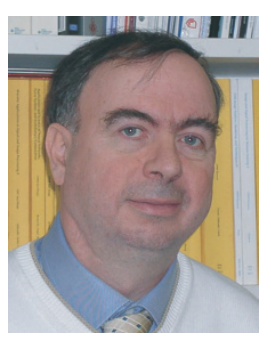
2001 he became a Researcher with IFAC-CNR (formerly IROECNR), Florence, Italy, where he currently participates in research activities concerning image quality definition and measurement, advanced methods for lossless and near-lossless remote sensing data compression, multiresolution image analysis and data fusion, and SAR image analysis and classification. He has been involved in several international research projects with ESA, CNES, and ASI on advanced data compression and image fusion algorithms for environmental applications. He is responsible for SAR image analysis and classification in a project funded by the Italian Space Agency.
He has coauthored over 20 papers published in international peerreviewed journals.

Stefano Baronti was born in Florence, Italy, in 1954. He received the Laurea degree in electronic engineering from the University of Florence, Florence, Italy, in 1980. After a period spent with the Italian Highway Company working on data collection and analysis, he joined the National Research Council of Italy (CNR) in 1985 as a Researcher of the "Nello Carrara" IFAC-CNR (formerly IROE-CNR), Florence, Italy. From 1985 to

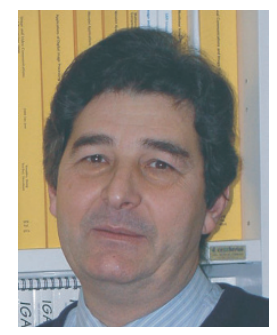
1989, he was involved in an ESPRIT project of the European Union aimed at the development of an automated system for quality control of composite materials through analysis of infrared image sequences. Later he moved toward remote sensing image processing by participating in and being the head of several projects funded by the Italian, French, and European space agencies. His research topics are in digital image processing and analysis aimed at computer vision and cultural heritage applications, data compression and image communication (including medical imaging), and opti$\mathrm{cal}$ and microwave remote sensing by synthetic aperture radar. $\mathrm{He}$ has coauthored about 40 papers published in international peerreviewed journals. He is a Member of IEEE Signal Processing Society and the IEEE Geoscience and Remote Sensing Society's Data Fusion Committee.

Massimo Bianchini was born in Florence, Italy. In 1999 he received the Laurea degree in electronic engineering from the University of Florence. From 1999 to mid-2003, he worked with the Microwave Remote Sensing Group, IFAC-CNR, Florence, Italy. His main interest was processing of synthetic aperture radar (SAR) imagery of the Earth for information extraction, in particular joint use of coherence and backscatters for change detection and land use mapping. In July 2003, he joined the Department of Electronics and Telecommunications, University of Florence, where he developed and assessed automated methods for coherence estimation from real-valued SAR image pairs. His present research interests are in preprocessing of SAR images for speckle reduction, fusion of optical and microwave data, and information extraction from hyperspectral image data.

Alessandro Mori received the Laurea degree "cum laude," in electronic engineering from the University of Florence, Florence, Italy, in 1995, and the Ph.D. degree in telecommunications and information engineering from the same university, in 1998. He is currently a Research Assistant at the Department of Electronics and Telecommunications of the University of Florence, working with the Applied Electro-

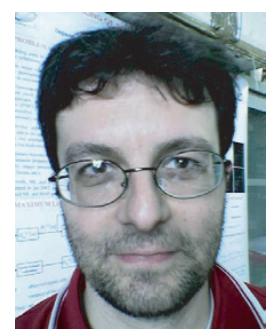
magnetism Laboratory. His research activities include active microwave remote sensing and numerical methods in electromagnetic scattering and radiation. He is currently investigating computationally efficient full wave methods for the analysis of electromagnetic scattering and radiation. 
Luciano Alparone obtained the Laurea degree in electronic engineering "summa cum laude" and the Ph.D. degree from the University of Florence, in 1985 and 1990, respectively. Since 1992, he has been with the Department of Electronics and Telecommunications of the University of Florence, firstly as an Assistant Professor, and since 2002 as an Associate Professor of electrical communications. In 1989 he was a Research

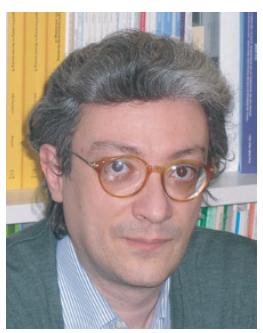
Fellow with the Signal Processing Division at the University of Strathclyde, Glasgow, UK. During spring 2000 and summer 2001, he was a Visiting Professor at the Tampere International Center for Signal Processing (TICSP), Tampere, Finland. His main research interests are lossless and near-lossless compression of remote sensing and medical imagery, multiresolution image analysis and processing, nonlinear filtering, multisensor image fusion, and analysis and processing of hyperspectral and synthetic aperture radar images. He coauthored fifty papers published in international peerreviewed journals and holds a patent on a procedure for progressive image transmission. 